\title{
Opinion Spreading with Mobility on Scale-Free Networks *
}

\author{
GUO Qiang(郭强) ${ }^{1}$, LIU Jian-Guo(刘建国 $)^{2,3 * *}$, WANG Bing-Hong(汪秉宏 $)^{2}$, ZHOU Tao(周涛 $)^{2,3}$, \\ CHEN Xing-Wen(陈兴文) ${ }^{1}$, YAO Yu-Hua(姚玉华) ${ }^{4 * * *}$ \\ ${ }^{1}$ School of Science, Dalian Nationalities University, Dalian 116600 \\ ${ }^{2}$ Department of Modern Physics, University of Science and Technology of China, Hefei 230026 \\ ${ }^{3}$ Department of Physics, University of Fribourg, CH-1700 Fribourg, Switzerland \\ ${ }^{4}$ School of Life Science, Zhejiang Sci-Tech University, Hangzhou 310018
}

\begin{abstract}
A continuum opinion dynamic model is presented based on two rules. The first one considers the mobilities of the individuals, the second one supposes that the individuals update their opinions independently. The results of the model indicate that the bounded confidence $\epsilon_{c}$, separating consensus and incoherent states, of a scale-free network is much smaller than the one of a lattice. If the system can reach the consensus state, the sum of all individuals' opinion change $O_{c}(t)$ quickly decreases in an exponential form, while if it reaches the incoherent state finally, $O_{c}(t)$ decreases slowly and has the punctuated equilibrium characteristic.
\end{abstract}

PACS: 89.75. - k, 87.23. Ge, 64.60. Cn

Recently, the study of complex systems has proven its significance in providing insight into many emerging interdisciplinary fields of science. ${ }^{[1-7]}$ In particular, great effort have been taken on the mathematical modelling of a rich variety of social phenomena, such as evolution of social structures, cooperation, opinion formation and spreading. ${ }^{[8-20]}$ In this Letter, we investigate the opinion spreading model defined on complex networks. In society, opinion evolution is determined by the interactions of the social members, which plays an important role in the opinion propagation. Individuals can be influenced by their neighbours or friends frequently. If two individuals are both supporters of a basketball team, they may like to discuss basketball and influence the opinions of each other; on the other hand, if two persons have opposite political views, they will hardly agree with each other and the exchange of their opinions can rarely arise. Axelrod ${ }^{[21]}$ proposed a model for the dissemination of culture to explain how different cultural islands could be generated from a local tendency to convergence. In his model, culture is expressed by integers. The number of features or dimensions is nothing but the number of components of a vector, and two persons interact if and only if they share at least one common feature. Starting from the Axelrod model, a number of simple agent-based models have been proposed. ${ }^{[21-24]}$

The opinion dynamics often starts from a random distribution of opinions, ${ }^{[22-24]}$ and evolves until reaching an equilibrium state characterized by the number of existing opinions. For instance, the basic Sz- najd model ${ }^{[25]}$ with random sequential updating always leads to a consensus (i.e. only one opinion survives) on a regular lattice of any dimension $D$. In the $\mathrm{KH}$ model, ${ }^{[24]}$ as $\epsilon$ departs from 0, many groups emerge, each of which is consisted of a few individuals sharing the same opinion. As $\epsilon$ becomes larger, a single group acquires more and more members, while the number of groups is reduced. Eventually, after arriving a critical value $\epsilon_{c}$, the whole system reaches a consensus state.

In reality, a social system should be viewed as a network where links are long-lived and facilitate many communication events. Recent empirical studies have demonstrated that some real-world networks can be treated as scale-free networks, ${ }^{[20]}$ such as the children friendship network, ${ }^{[29]}$ and scientific collaboration network. ${ }^{[30]}$ Recent works on the topic of scalefree networks have been driven largely by the studies on network dynamics, [31-45] optimization $^{[46-49]}$ and evolution. ${ }^{[50-65]}$ In order to make the opinion evolution more realistic, we model the opinion spreading on a scale-free network. ${ }^{[66]}$ A total fraction $p$ of the sites are occupied by individuals who can move to the empty sites under certain condition.

The individuals can exchange their opinions with their neighbours if the difference of their opinions is smaller than a 'bounded confidence' $\epsilon$, which measures the tolerance for dissent or the openness to different opinions. If the difference of their opinions is greater than $\epsilon$, one will move to an empty site in its neighbourhood.

* Supported by the National Basic Research Programme of China under Grant No 2006CB705500, the National Natural Science Foundation of China under Grant Nos 10635040, 10532060, 70571074 and 10472116, the Special Research Funds for Theoretical Physics Frontier Problems (A0524701), the President Fund of Chinese Academy of Sciences, the Specialized Research Fund for the Doctoral Programme of Higher Education of China, and the Research Fund of the Education Department of Liaoning Province (20060140).

**Email: liujg004@ustc.edu.cn

***Email: yaoyuhua2288@163.com 

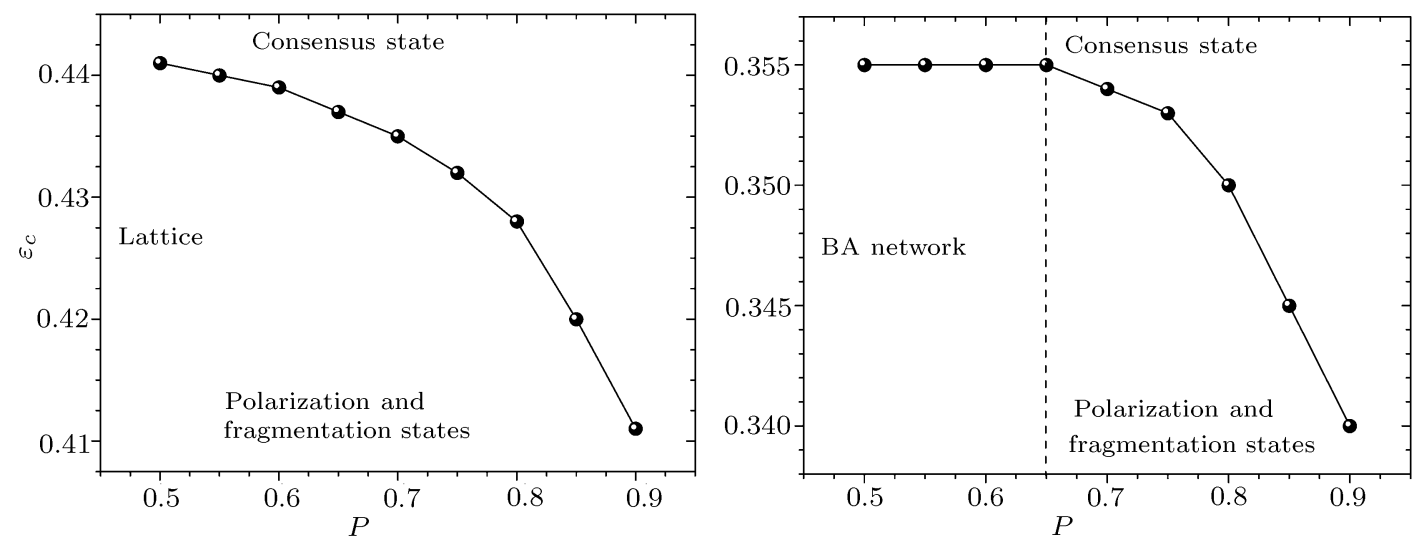

Fig. 1. Consensus phase diagram of the current model on BA networks and lattice with $N=1024$. When $\epsilon$ is larger than $\epsilon_{c}$, all individuals can reach the consensus state. When $\epsilon<\epsilon_{c}$, they would reach the incoherent state. All the data are averaged over 50 independent runs.

The model starts on a network with $N$ sites, each of which is occupied by an individual with probability $p$. A random number in $[0,1]$ is given to each individual, which represents its initial opinion. For individual $i$, randomly select one of its neighbours, say $j$. If $\left|o_{i}(t)-o_{j}(t)\right|<\epsilon$, both $i$ and $j$ have no willing to move in this time step and they will update their opinions in the following way:

$$
\begin{aligned}
& o_{i}(t)=o_{i}(t)-a_{i j}(t)\left(o_{i}(t)-o_{j}(t)\right), \\
& o_{j}(t)=o_{j}(t)+b_{i j}(t)\left(o_{i}(t)-o_{j}(t)\right),
\end{aligned}
$$

where $a_{i j}(t)$ and $b_{i j}(t)$ are two random numbers in $[0,0.5]$. When $\left|o_{i}(t)-o_{j}(t)\right|>\epsilon$ and there exists at least one empty site in $i$ 's neighbourhood, $i$ will move to a randomly selected empty neighbouring site.

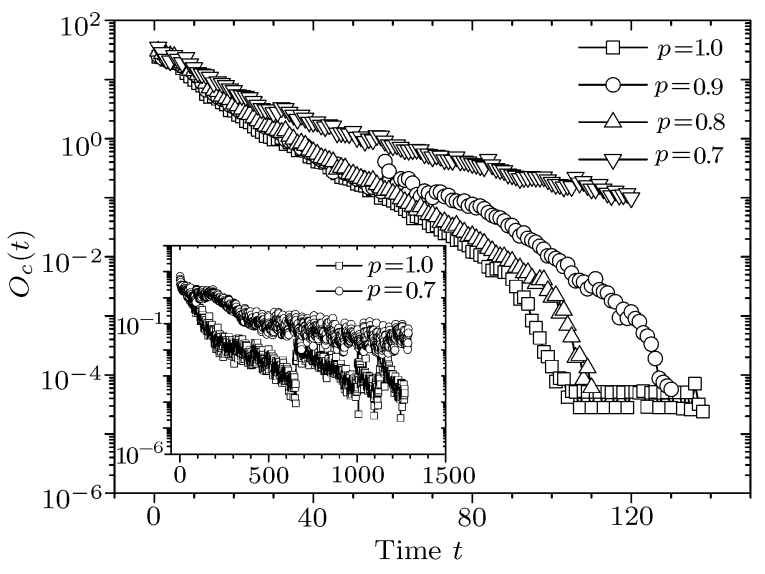

Fig. 2. $O_{c}(t)$ vs. $t$ for different $\epsilon$ and $p$.

Let $O_{c}(t)$ denote the total opinion change of all individuals at time step $t$ :

$$
O_{c}(t)=\sum_{i}\left\{o_{i}(t)-o_{i}(t-1)\right\}
$$

The dynamics is terminated when $O_{c}(t)<T_{c}$, where $T_{c}$ a threshold given in advance. The numerical simulations are implemented on the BA network and the terminal condition is set as $T_{c}=10^{-10}$.

We find that when $p \neq 1$ and $\epsilon$ is close to 0.5 , the number of groups is remarkably decreased. Slowly increasing $\epsilon$, opinion groups will collide and merge, annihilate and vanish, and eventually there is only one group survives after $\epsilon$ exceeds a critical point $\epsilon_{c}$. Accordingly, we can say there is a phase transition, in which the number of different groups can be regarded as the order parameter, whose value determines the state of the system. If the value is 1 , the system is in consensus state, otherwise in incoherent state (with 2 groups we call polarization state, while for $>2$ groups we call fragmentation state). In this way, we can calculate various $\epsilon_{c}$ for different values of $p$, and then draw a phase curve which separates the consensus state and the incoherent state. It is worth stressing that when the individuals have the mobility on the network, the value of $\epsilon_{c}$ reaching consensus is remarkably decreased. The phase diagram of $p$ vs $\epsilon_{c}$ is shown in Fig. 1, which quantifies our above analysis. From Fig. 1, one can see that both the bounded confidences $\epsilon_{c}$ of the BA network and lattice are smaller than 0.5, and both of $\epsilon_{c}$ decrease as $p$ increases, which indicates the fact that when the population density $p$ increasing, all of the individuals reach consensus state more easily. It should also be emphasized that the $\epsilon_{c}$ of the BA network is smaller than that of lattice and began to sharply decrease after $p=0.65$. The simulations also indicate that when $p<0.5$ (not shown in Fig. 1), $p$ can only influence the opinion spreading speed and has little effect on $\epsilon_{c}$. Figure 2 shows the change process when $\epsilon=0.5$ corresponding to the consensus state, from which one can find that the sum of opinion changes $O_{c}(t)$ decrease exponentially. The inset shows the one when $\epsilon=0.2$ corresponding to the incoherent state, where $O_{c}(t)$ decreases slowly and 
has the punctuated equilibrium characteristic, which may be caused by the debates among different factions.

In summary, when the mobilities of the individuals are taken into account, an opinion spreading model on BA network is presented. The opinion spreading process is based on the interaction structure of individuals, when their opinions lie into the bounded confidence $\epsilon$, they can updated their opinions independently. The numerical results indicate that bounded confidence $\epsilon_{c}$ of the model on BA network is smaller than the one of lattice, and the convergence speed is more quickly. It is worth stressing that $O_{c}(t)$ decreases exponentially in the case that the system can reach the consensus state, while if it reaches the incoherent state finally, it decreases slowly and has the punctuated equilibrium characteristic. Future works will focus on the opinion dynamics on real social networks with assortative coefficient.

The authors thank Dr Ming Zhao for her comments and suggestions.

\section{References}

[1] Candia J 2007 Phys. Rev. E 75026110

2[] Stauffer D et al 2005 Phys. Rev. E 72046128

[3] Lorenz J 2007 arXiv:0707.1762

[4] Rosvall M et al 2007 arXiv:0708.0368

[5] Weidlich W 1991 Phys. Rep. 2041

[6] Weidlich W 2000 Sociodynamics: A Systematic Approach to Mathematical Modelling in the Social Sciences (Amsterdam: Harwood Academic)

[7] Oliveira S M de et al 1999 Non-Traditional Applications of Computational Statistical Physics (Stuttgart: Teubner)

[8] Sznajd-Weron K et al 2000 Int. J. Mod. Phys. C 111157

[9] Castellano C et al 2000 Phys. Rev. Lett. 853536

[10] Aleksiejuk A et al 2002 Physica A 310260

[11] Klemm K et al 2003 Phys. Rev. E 67026120

[12] González M C et al 2004 Int. J. Mod. Phys. C 1545

[13] Suchecki K et al 2005 Phys. Rev. E 72036132

[14] Kuperman M N 2006 Phys. Rev. E 73046139

[15] González M C et al 2006 Eur. Phys. J. B 49253

[16] Candia J 2006 Phys. Rev. E 74031101

[17] Watts D J 1999 Small Worlds (Princeton, NJ: Princeton University Press)

[18] Zhou T et al 2007 Phys. Rev. E 76046115

[19] Zhou T et al 2006 Phys. Rev. E 74056109

[20] Albert R et al 2002 Rev. Mod. Phys. 74 47; Dorogovtsev S N et al 2003 Evolution of Networks (New York: Oxford University Press);
Strogatz S 2003 SYNC-How the Emerges from Chaos in the Universe (New York: Hyperion)

Wang X F 2002 Int. J. Bifurcat. Chaos 12885

Newman M E J 2003 SIAM Rev. 45 167; Boccaletti S et al 2006 Phys. Rep. 424175

Newman M E J et al 2006 The Structure and Dynamics of Networks (Princeton, NJ: Princeton University Press)

[21] Axelrod R 1997 J. Conflict Resolution 41203

[22] Sznajd -W K et al 2000 Int. J. Mod. Phys. C 111157

[23] Deffuant G et al 2000 Adv. Complex Systems 3 87; Weisbuch G 2004 Eur. Phys. J. B 38339

[24] Hegselmann R et al 2002 J. Arti. Soc. Social Simul. 5 (3) (paper 2 jasss.soc.surrey.ac.uk)

[25] Stauffer D 2003 AIP Conf. Proc. 690147

[26] Ben-Naim E et al 2003 Physica D 183190

[27] Fortunato S et al 2005 cond-mat/0501730

[28] Fortunato S 2005 Int. J. Mod. Phys. C 16259

[29] $\mathrm{Hu}$ B et al 2005 Physica A $\mathbf{3 5 3} 576$

[30] Li M et al 2005 Physica A $\mathbf{3 5 0} 643$

[31] Tadić B et al 2004 Phys. Rev. E 69036102

[32] Zhao L et al 2005 Phys. Rev. E 71026125

[33] Yan G et al 2006 Phys. Rev. E 73046108

[34] Yin C Y et al 2006 Phys. Lett. A 351220

[35] Pastor-Satorras R and Vespignani A 2001 Phys. Rev. Lett. 863200

[36] Yan G et al 2005 Chin. Phys. Lett. 22510

[37] Zhou T et al 2006 Prog. Nat. Sci. 16452

[38] Motter A E and Lai Y -C 2002 Phys. Rev. E 66065102

[39] Goh K I et al 2003 Phys. Rev. Lett. 91148701

[40] Zhou T and Wang B -H 2005 Chin. Phys. Lett. 221072

[41] Zhou T et al 2005 Phys. Rev. E 72016139

[42] Zhao M et al 2005 Phys. Rev. E 72057102

[43] Zhou T et al 2006 Phys. Rev. E 73037101

[44] Duan W Q et al 2005 Chin. Phys. Lett. 222137

[45] Fan J et al 2005 Physica A 355657

[46] Valente A X C N et al 2004 Phys. Rev. Lett. 92118702

[47] Paul G et al 2004 Eur. Phys. J. B 38187

[48] Liu J G et al 2005 Mod. Phys. Lett. B 19785

[49] Liu J G et al 2006 Mod. Phys. Lett. B 20815

[50] Zhou $\mathrm{T}$ et al 2005 Phys. Rev. E 71046141

[51] Andrade J S et al 2005 Phys. Rev. Lett. 94018702

[52] Dorogovtsev S N et al 2001 Phys. Rev. E 63062101

[53] Holme P and Kim B J 2002 Phys. Rev. E 65065107

[54] Newman M E J 2002 Phys. Rev. Lett. 89208701

[55] Dorogovtsev S N et al 2002 Phys. Rev. E 65066122

[56] Ravasz E and Barabási A -L 2003 Phys. Rev. E 67026112

[57] Jiang P Q et al 2005 Chin. Phys. Lett. 221285

[58] Wang W X et al 2005 Phys. Rev. E 72046140

[59] Wang W X et al 2005 Phys. Rev. Lett. 94188702

[60] Zhu C P et al 2004 Phys. Rev. Lett. 92218702

[61] Guo Q et al 2006 Physica A 371841

[62] Liu J G et al 2006 Physica A 366578

[63] Liu J G et al 2006 Physica A 371861

[64] Liu J G et al 2007 Physica A 377302

[65] Liu J G et al 2006 Chin. Phys. Lett. 23746

[66] Barabási A-L and Albert R 1999 Science 286509 